
\title{
$\angle$ Research Square \\ Social Synchronization During Joint Attention in Children with Autism Spectrum Disorder
}

\section{Qinyi Liu}

Peking University Sixth Hospital https://orcid.org/0000-0001-9953-5184

Qiandong Wang

Peking-Tsinghua Center for Life Sciences

Xue Li

Peking University Sixth Hospital

\section{Xiaoyun Gong}

Peking University Sixth Hospital

\section{Xuerong Luo}

Department of Psychiatry, the Second Xiangya Hospital

\section{Tingni Yin}

Peking University Sixth Hospital

Jing Liu

Peking University Sixth Hospital

Li Yi ( $\square$ yilipku@pku.edu.cn )

Peking University https://orcid.org/0000-0003-4117-8828

\section{Research}

Keywords: social synchronization, joint attention, autism spectrum disorder, eye movement, crossrecurrence quantification analysis, gaze-shift behaviors

Posted Date: October 9th, 2020

DOI: https://doi.org/10.21203/rs.3.rs-88767/v1

License: (c) (i) This work is licensed under a Creative Commons Attribution 4.0 International License. Read Full License 


\section{Abstract}

Background: This study examined the social synchronization in children with autism spectrum disorder (ASD) when responding to others' joint attention. Social synchronization refers to an individuals' temporal coordination during social interactions, which has been found to play a crucial role in social development. Deficient joint attention has been repeatedly found in individuals with autism spectrum disorder, and previous studies have demonstrated various explanations about it. In a more recent perspective, joint attention could be associated with spontaneous social motor synchronization, and it is possible to explore social synchronization as a pathway to understanding the impairments of joint attention in children with ASD.

Methods: Forty-one children aged 5 to 8 with ASD and 43 age-matched typically developing (TD) children watched a video to completed the response to joint attention (RJA) tasks, during which their gaze data were collected. The synchronization of gaze-shift behaviors between children and the female model in the video was measured with the cross-recurrence quantification analysis (CRQA).

Results: We found that children with ASD had the ability, to some extent, to synchronize their gaze shifts with the female model in the video during RJA tasks. However, compared to the TD children, children with ASD displayed lower levels of synchronization and longer latency in this synchronized behavior.

Limitations: The RJA task in our study was not a real social interaction task but rather a one-way interaction. Social interaction between two persons through live video or a natural scenario should be further explored. Besides, additional work is necessary to determine whether our findings generalize to individuals across the full autism spectrum.

Conclusion: These findings provide a new avenue to deepen our understanding of the impairments of joint attention in children with ASD. Notably, the analytic method can be further applied to explore the social synchronization of numerous other social interactive behaviors in ASD. Additionally, the impairment of social synchronization may be a new implicit indicator for the evaluation of autism and can be utilized to screen children with ASD along with other indicators.

\section{Background}

Social synchronization refers to an individuals' temporal coordination during social interactions[1]. Particularly, it includes various social communicative processes such as joint attention, imitation, turntaking, non-verbal social communicative exchanges, affect sharing, and engagement [2]. Notably, social synchronization has been found to play a crucial role in social development [3]. For instance, interactional synchronization in mother-infant interactions is imperative for the development of social relations and language learning[4, 5]. Additionally, social synchronization has been found to be associated with children's social responsiveness [6], social rapport[7, 8], social perception[9], cooperation[10-12], positive interpersonal relationships[13,14], as well as with a better understanding of the thoughts and actions of others[15]. Some researchers have proposed that social synchronization could be a potentially significant 
pathway to understanding the underlying mechanisms of impairments in social functioning, such as joint attention, cooperation, and comprehending the intentionality of others $[16,17]$. This study aimed to explore social synchronization during joint attention in typically developing (TD) children as well as in children with autism spectrum disorder (ASD).

Joint attention (JA), typically emerging between 6 and 9 months in infants, is a social skill which shares the focus of attention with another individual to a common point of reference. JA involves following (i.e., responding to joint attention, RJA) and directing (i.e., initiating of joint attention, IJA) another person's focus of attention towards a target $[18,19]$. Joint attention is a vital social skill, which is found to be predictive of subsequent development of language [20-23] and social skills [19, 24, 25]. It is also associated with development of the theory of mind [26], emotional regulation [27], and cognitive development $[24,28,29]$.

Deficient joint attention has been generally found in individuals with ASD, a neurodevelopmental disorder characterized by impairments of social interaction, communication and restricted, repetitive patterns of behavior and interests [30]. Previous studies have identified that children with ASD demonstrate fewer responses to others' joint attention beginning at an early age [31-34]. Although other studies reflect that children with ASD exhibit an intact ability to follow the gazes of others, they display a shorter period of engagement in the target of others' attention [35], and slower correct gaze shifts from the person to the attended object compared to TD children $[32,36]$. Why do children with ASD display atypical ability to respond to joint attention? One possible explanation could be attributed to the fact that children with ASD have difficulties in attending to and interpreting referential cues in social contexts, such as an agent's eye gaze or head turn to understand an agent's intentions [37,38]. Meanwhile, they could also demonstrate diminished monitoring and responsivity to social cues that signal goal-directedness [39]. Besides, their impairments in joint attention could be accounted for by a lack of social motivation, atypical resistance to distraction, enhanced parallel perception, and atypical execution of volitional actions such as gaze dyspraxia [40,41]. In a more recent perspective, RJA could be associated with spontaneous social motor synchronization, a type of social synchronization. To respond to joint attention, it is necessary for children to initially attend to the social cue while also possessing the motivation to respond to it. Considering the role of social motor synchronization in constructing social bonds with other people and responding to social cues [3] as well as autistic people's difficulty in synchronizing their movements with another person $[6,16,42-44]$, it is reasonable to explore social synchronization as a pathway to understanding the impairments of RJA in children with ASD.

To examine the social synchronization of children with ASD during joint attention, we used the RJA task adapted from Senju and Csibra [45]. In this task, we presented children with a video depicting a female model turning toward one of the two objects, and recorded children's eye movements when watching the video to examine their gaze-following behavior. Considering that social interaction is essentially a continuous process, we analyzed all the trials as a whole continuous process through the use of crossrecurrence quantification analysis (CRQA) to examine the synchronization of gaze-shift behaviors between children and the female model in the video. Notably, CRQA can quantify how similarly two 
observed data series unfold over time $[46,47]$. This is not to mention that it also reflects the degree of synchronization as well as the level of delay in synchronization between pairs of people [15]. This method has been employed to measure different behavioral coordinations between individuals, such as the shared postural configurations of two people engaged in a conversation [48], and the looking behaviors of infants and their caregivers when actively playing with toys [49].

In this study, we applied the cross-recurrence quantification analysis to measure the synchronization of gaze-shift behaviors between children and the female model in the video during the RJA tasks. Previous research has identified that children with ASD exhibited the intact ability to follow gaze, but there was reduced time spent on the attended objects and slower correct gaze shifts [32, 35, 36]. Accordingly, we expected that children with ASD in our study would demonstrate the ability to synchronize their gaze shifts with the female model in the video during RJA tasks. Notably however, their latency in this synchronized behavior would be longer and their level of synchronization would also be lower than TD children.

\section{Method}

\section{Participants}

A total of 84 children aged 5 to 8 participated in the study, including 41 children with ASD (37 males, 4 females) and 43 TD children (38 males, 5 females). One child with ASD and three TD children were excluded from analysis due to poor eye movement data quality (see "Data Analysis" section for details), resulting in 40 children with ASD ( 36 males, 4 females) and 40 TD children ( 36 males, 4 females) in the final sample. Children with ASD were recruited from Peking University Sixth hospital, and had been diagnosed by two professional child psychiatrists according to DSM-5 [30]. The diagnosis was confirmed using the Chinese version of the Autism Spectrum Quotient - Children's Version (AQ-Child; [50]. The exclusion criteria for the ASD group were as follows: (a) AQ-Child score < 73 [51]; (b) having severe psychiatric disorders or physical diseases; (c) having uncorrected hearing or visual impairments (d) unable to cooperate with the eye-tracking test. The TD sample was recruited from local communities by advertisements online as well as from local primary schools in Beijing. The two groups were matched by chronological age and non-verbal IQ score (measured by Raven's Test, see Table 1). Detailed descriptions of participant characteristics can be found in Table 1.

\section{Ethical considerations}

This study was approved by the Ethical Committee in Peking University Sixth Hospital and conducted based on the 1964 Declaration of Helsinki. All parents and children gave written consent before the onset of the experiment.

\section{Stimuli}


Children were asked to watch a video (lasting for $84 \mathrm{~s}$ ) depicting a female model sitting behind a table where two identical objects were placed (Figure 1). The video consists of two rounds, which features different objects and each round includes four responding to joint attention (RJA) tasks. At the beginning of each round, the model's face is covered by a cartoon accompanied with a voiceover, 'Look!' so as to attract the child's attention. The cartoon then disappears, revealing the model's face. The model looks straight into the camera, smiling for about $5 \mathrm{~s}$. Then, the four RJA tasks/trials are presented in random order. There are two different cues (only gaze shifting, gaze and head shifting simultaneously) in the RJA tasks. Each cue appears twice in each round, once to the left and once to the right (the positions are counterbalanced across trials). In each task/trial, the model shifts gaze which is either accompanied by the gesture of turning her head towards one of the two objects after looking straight into the camera for about $300 \mathrm{~ms}$, or not doing so. Then, the model continues to look at the attended object for $4 \mathrm{~s}$ and turns back to look straight at the participant for $3 \mathrm{~s}$ at the end of the task where a cartoon character appears beside the attended object.

\section{Procedure}

After arriving at the lab, the parents sign the consent form and complete the AQ-child questionnaire. Then, the child is taken to the eye-tracking lab and is seated in the chair at a distance of $60 \mathrm{~cm}$ in front of a 17inch LCD monitor (1680×1050 pixels resolution). A 5-point calibration procedure is then conducted to calibrate their eye movements. During the calibration process, the child is instructed to fixate on the five red calibration points which appear sequentially in the center and four corners of the screen. The calibration is repeated to many times necessary until both eyes achieve good mapping on all five calibration points (smaller than a $2^{\circ}$ visual angle). After the calibration, the child is asked to watch the video and no further instruction is provided. The two rounds of the video contains a total of eight RJA tasks/trials which are played randomly. Eye movement data were recorded throughout the entire experiment.

\section{Measures}

The Autism Spectrum Quotient - Children's Version (AQ-Child; [50] is a parent-report questionnaire, which consists of 50 items created to measure autistic traits in children between the ages of 4 and 11. Here, we used the Chinese version of the AQ-Child [51] to assess each of the five domains: (1) social skill (2) attention switching (3) attention to detail (4) communication (5) imagination. Parents rated the extent to which they agree or disagree with the statements about their child on a 4-point scale (0 represented definitely agree, 1 slightly agree, 2 slightly disagree, and 3 definitely disagree). Higher scores of AQ-Child denote higher levels of autistic traits. The AQ score has been shown to have high sensitivity $(96 \%)$ and specificity (90\%) at a cut-off of 73 and has good test-retest reliability (92\%) [51]. In this sample, the mean AQ score of the ASD group was $85.8 \pm 8.6$.

\section{Data Analysis}

\section{Data preprocessing}


Gaze data were recorded using a Tobii T120 eye tracker and Tobii Studio software (Tobii Technology, Stockholm, Sweden). Three rectangular areas of interests (AOIs) were defined: one covered the female model's face while the other two covered the two objects (see Figure 1). Raw gaze data (i.e., sample data) were extracted from the Tobii Studio software. Notably, trials with more than $50 \%$ invalid gaze data (defined as validity codes $>1$ from Tobii raw data) were excluded from the analysis. To ensure the data's quality, we excluded children with fewer than six valid trials after trial rejection from further analyses.

\section{Cross-Recurrence quantification analysis of two gaze data streams}

Cross-recurrence quantification analysis (CRQA) (Xu et al., 2020) was applied to quantify coordinated attention between children and the female model, and to measure their temporal coupling with different degrees of time lags. The precise timing information for the gaze data streams of the children and the female model can be depicted graphically in what we refer to as the 'cross-recurrence plot.' Here, the gaze data of one child and the female model was used during RJA tasks as a reference to explain the method. As depicted in Figure 2, the horizontal dimension represents the child's gaze stream over time while the vertical dimension represents the female model's gaze stream over time. We calculated synchrony during the RJA trials (Figure 1c \& 1d). Additionally, the central diagonal line in the cross-recurrence plot provides a measure of synchrony when simultaneously looking to the same AOI. Specifically speaking, the colored pixels on the diagonal line indicate that the eyes of the child and the female model were fixated on the same object or they were directly looking at each other (when the female model was looking directly and the child was looking at the model's face). The white pixels demonstrate that the child and the female model were not fixated on the same AOI. Meanwhile, the pixels in other diagonal lines that are parallel to the central diagonal line of the plot reflect time-shifted recurrences between the gaze of the child and the female model. These pixels depict whether the child and the female model were fixated on the same AOI or if they expressed a mutual gaze but with some delay (time unit: 1/120 seconds based on the sample rate of the eye tracker). To quantify the patterns of synchronization in these two data streams, we generated the cross-recurrence lag profiles through computing the percentage match (or percentage recurrence, \%REC) along all the diagonal lines in the cross-recurrence plot. These profiles reflect the synchronization between two data streams at a different time lag. It should be noted that the current task was not a real social interaction task and the female model could not respond to the children's gaze, thus the percentage recurrence along the lower right diagonal lines was reported.

Three statistical analyses were applied to examine whether children with ASD exhibited atypical social synchronization patterns with the female model compared to TD children. Particularly, the synchronization patterns of the ASD and the TD groups were compared with chance separately, and then with each other. Then, we assessed whether group membership could be predicted from the percentage recurrence at different time lags through using a machine learning algorithm to validate the above inferential statistical analyses. All the detailed descriptions of the methods can be found in the supplementary materials online.

\section{Results}




\section{Synchronization between the eye movements of the children and the female model}

Figure 3 depicts the average percentage recurrence in the ASD and TD group pairings for each time lag, with " 0 " indicating simultaneous fixations to the corresponding AOls (including mutual gaze). The curve to the right of " 0 " reflects that the female model's looks to an $A O l$ that was followed by a look to the same $\mathrm{AOI}$ by the child within the defined lag. The black line below depicts a lag time at which the child and the female model look at the same AOI at above-chance levels. As demonstrated in Figure 3 , both groups were not randomly looking around the video, which reflected in their above-chance performance of gaze following. The ASD group began following the female model's look to the same AOI at above-chance levels $800 \mathrm{~ms}$ after the model shifted her gaze or turned her head, Meanwhile, the TD group immediately followed the female model's orientation as the model shifted her gaze or turned her head.

We then compared the synchronization patterns of children with the female model between the ASD and the TD groups. The baselines of the two groups differed (Figure 3) due to the different gaze data loss rate (when the child looked at blank spaces or looked off the screen or blinked), making the two groups incomparable. To resolve this issue, we subtracted each child's percentage recurrence at different time lags by his/her baseline which was generated through shuffling the temporal order of each child's eyemovement sequence 1000 times and calculating the average recurrence with the female model.

Figure 4 illustrates the difference of the percentage recurrence between the two groups with the adjusted baseline. The black line below indicates the lag time at which the average percentage recurrence in the ASD and TD group were significantly different. The average percentage recurrence in the ASD group was lower than that of the TD group at the lag time between 0 and $2883 \mathrm{~ms}$. This is not to mention that the maximum recurrence between the model and children of the two groups was different as well, with $6.57 \%$ in the ASD group and $8.59 \%$ in the TD group. The lag time at which their eye movements overlap the most, is $3292 \mathrm{~ms}$ in the ASD group and $2025 \mathrm{~ms}$ in the TD group.

\section{The classification performance of the RJA tasks}

The support vector machine (SVM) was used with linear kernel [52], which has been widely utilized in psychiatry research as a classification algorithm to evaluate the prediction performance. Overall, classification performance resulted in $60 \%$ accuracy. The sensitivity and specificity were $63 \%$ and $58 \%$, respectively. The area under the curve (AUC) of the receiver operating characteristic curve (ROC) was 0.63 . The permutation test reflected that the prediction was higher than the random level, $p=0.042$.

\section{Discussion}

In this study, we explored the atypical patterns of social synchronization during response to joint attention in children with ASD through utilizing the analytical method of cross-recurrence quantification analysis (CRQA). Consistent with our hypotheses, children with ASD was identified to possess the ability, to some extent, to synchronize their gaze shifts with the female model in the video during the RJA tasks. 
Compared to the TD children however, they displayed lower levels of synchronization and longer latency in this synchronized behavior.

First, we discovered that when compared with the baselines, both groups were looking at the same AOI with the female model at above-chance levels, indicating that they were not looking randomly during the RJA tasks. This further suggests that children with ASD can, to some extent, respond to social cues such as gaze shift and head turn. This finding is consistent with previous studies which demonstrate that children with ASD had the ability to follow gaze during RJA tasks [35, 36]. Upon comparing the two groups however, we found that the level of gaze-shift synchronization in children with ASD was still lower than that in the TD children. This suggests that the ability to synchronize the gaze shifts with social partners in children with ASD, although above the chance level, is still poorer than TD children. The level of synchronization here was determined by the length of time children spent on the same AOls where the female model also looked. Here, we assume that that the level of synchronization could be influenced by two factors: gaze following accuracy (the children follow the female model's gaze to the attended object), and the duration of fixations at the female model's face or the attended objects. Vivanti et al. [34] has found that children with ASD demonstrated decrease attention to the model's face when the model looked straight during RJA tasks. Bedford et al. [35] and Falck-Ytter et al. [36] have also noted that the time they spent on the attended objects was shorter than that in the TD group despite the intact gaze following accuracy in the ASD group. These factors could all contribute to the low level of synchronization in the ASD group during the RJA tasks. The reason why children with ASD allocated less attention to the attended object could be attributed to their difficulty in understanding communicative meaning of the social referential cues such as gaze shift [35]. Based on the previous study, the eyelooking time positively correlated with the subsequent attention on the object in TD children, but this is not the case in children with ASD. It reflected that these children were impaired in understanding the social meaning of gaze and could not actively utilize gaze cues to determine their attention like the TD children [53]. Ultimately, our results suggest deficient synchronization performance during RJA in children with ASD, which may result in an insufficient process of social information during social interaction such as joint attention.

Second, a delayed social synchronization was identified during joint attention in ASD. Unlike TD children who promptly demonstrated gaze-shift synchronization in responding to the female model's gaze shift, children with ASD displayed a longer latency to respond to it. In addition, the lag time at which the ASD group had the best synchronization performance was longer than that in TD group. Such a longer latency in gaze-shift behavior of children with ASD was also identified in some previous studies concerning RJA [32]. These results together suggest that children with ASD had an impairment of synchronization in the dimension of time, and they were not as sensitive to social cues such as gaze shifts as compared with TD children. As responding to the social cues in a timely manner can help people effectively capture some useful but ephemeral information in social interactions, it is vital to explore the latency in social synchronization as well as the motivating reasons. Here, it was speculated that this latency could be accounted for by several factors. First, children with ASD may have difficulties in interpreting social cues $[37,38]$, so they need more time to process these cues before responding to them. Second, previous 
studies have found that children with ASD are resistant to distraction and may need more time to disengage from the model's face or the attended object $[54,55]$. Third, some research has suggested that children with ASD also suffer from gaze dyspraxia, suggesting that they have eye-motor difficulties in looking where another person is pointing or if they are asked to look [40]. As a result, it may be the incapability of gaze movement rather than the deficit in intentionality to respond to the gaze shift that underlies the delayed latency of gaze-shift synchronization. The combination of these three factors could contribute to the delay in gaze-shift synchronization of children with ASD, and more future evidence is required to support this conclusion.

Third, we further reviewed whether group membership could be predicted from the percentage recurrence through using a machine learning method as a supplementary analysis to verify the traditional analytical methods applied in this study. Generally, it was found that the overall classification performance resulted in $60 \%$ accuracy which differed from chance. This implies that the performance of gaze-shift synchronization could be a potential indicator to differentiate children with ASD from TD children. Meanwhile however, considering the low sensitivity and specificity of the single indicator, it is necessary to combine other characteristic indicators of ASD, such as the geometric preference [56], the abnormality in face scanning [57, 58], and facial affect recognition [59]. This is especially crucial in building a more effective and reliable model to effectively screen children with ASD.

In this study, it was found that children with ASD exhibited impairment of social synchronization during joint attention. However, certain questions still remain concerning the relationship between the impairments of social synchronization and joint attention in ASD. Here, it has been proposed that there are three possible relationships between them. First, the impairment of social synchronization could be an underlying mechanism of impairments of joint attention in children with ASD. Notably, some researchers suggest that the impairment of social synchronization ability during the neonatal period may disrupt the development of joint attention between the 6 to 9 month period. It should be noted that this could lead to difficulties in building social and emotional bonds with caregivers, as well as in understanding and responding to social cues [3]. Second, there may be some common underlying mechanisms of social synchronization and joint attention. For instance, both require the perception and information processing from others' actions as well as the social motivation to attend to and respond to the social interactions of others. Third, joint attention could play an imperative role in social synchronization. With the ability to synchronize with others' movements, people need to attend to the rhythms of others while also understanding or even inferring their intentions through the ability of joint attention. Generally speaking, it is difficult to reach a unanimous conclusion about their relationship in the current study, and more future evidence is needed to reach a conclusion.

In our study, we explored the impairment of joint attention from a new perspective -social synchronization: through a classical RJA paradigm and attained direct evidence concerning the impairment of synchronization in joint attention. In previous studies, the gaze following of children with ASD was demonstrated in discrete experimental trials without considering social interaction to be a continuous adaptational and coordinated behavior. Furthermore, these studies also ignored the 
redirection of the attention to the partner's face to form the mutual gaze after gaze following, which is suggested to be a critical component of joint attention. These limitations suggest a serious gap in obtaining a comprehensive understanding of how children with ASD coordinate and synchronize visual attention. This is especially with others in the complex social contexts of everyday life with moving heads and eye gaze in a continuous manner. Our study bridged this gap through using the CRQA method to investigate the continuous adaptation of the attention of autistic children to the visual attention of others. Through this analytic method, a more comprehensive understanding of social synchronization can be obtained compared with previous studies about social motor synchronizations $[3,6,16]$. In the dimension of time, we could explore the latency of gaze-shift synchronization during the RJA tasks. In the dimension of synchronization level, we will not only simultaneously understand the synchronization performance but also obtain crucial information of this at different lag times. Moreover, this method can be further applied in other social interactions such as social communicative exchanges, cooperation and imitation, and provide a new pathway to understanding the social defects in children with ASD.

\section{Limitations}

Five main considerations emerged from the current findings. First, the RJA task in our study was not a real social interaction task but rather a one-way interaction, that is, the child responded to the female model in the video. Social interaction between two persons through live video or a natural scenario that can reflect real social situations in daily life should be further explored in the future investigations.

Second, social synchronization includes various social interactive behaviors, and we solely focused on social synchronization during joint attention. Social synchronization in many other aspects of social interactions, such as face-to-face communication, imitation, turn-taking, cooperation, and joint actions, should be further examined so as to better grasp the social defects in ASD.

Third, previous studies have identified increased interpersonal neural synchronization in the frontal cortex during cooperative interactions in ASD and TD people, and weaker neural synchronization could predict poorer cooperative performance $[60,61]$. Here, the behavioral performance of social synchronization during joint attention was analyzed. Future studies could combine the examination of the underlying neural mechanisms of social synchronization during joint attention along with other social behaviors.

Fourth, the female model in our video is a stranger to the children. Considering the relationship between the dyads may affect the social interaction [62], it is necessary to consider different types of partners, such as parent-child, peers, and teacher-student, when examining the social interactive behaviors of children with ASD in future investigations.

Lastly, children with ASD who participated in our study had a relatively high IQ. However, it should be noted that, but in fact, there are also many children with ASD who have intellectual disabilities. So the results that were identified here can not be applied for all the children with ASD. Notably, children with ASD who also have intellectual disabilities should be considered for future studies. Besides, children 
between the ages of 5-8 were also studied so other age groups, especially those of younger ages, should be assessed for early screening.

\section{Conclusion}

We examined social synchronization in children with ASD as a new pathway to better understand their impairment of joint attention. However, joint attention is just one of the many behaviors that involve social synchronization. Our analytic method can be applied in future studies so as to explore the social synchronization of many other social interactive behaviors in ASD, and can offer behavioral evidence which can be combined with the study in neural synchronization. Additionally, the impairment of social synchronization may be a new implicit indicator for the evaluation of autism and can be utilized to screen children with ASD along with other indicators. Besides, such an understanding of the synchronizing impairments of different social interactions can reveal significant implications for developing new training protocols to improve interpersonal interactions and serve as an indicator for more efficient assessments in ASD interventions.

\section{Abbreviations}

ASD

Autism spectrum disorder; TD:Typically developing; JA:Joint attention; RJA:Response to joint attention; IJA:initiating of joint attention; CRQA:Cross-recurrence quantification analysis; AQ-Child:Autism Spectrum Quotient - Children's Version; AOI:Area of interests; \%REC:percentage recurrence; SVM:Support vector machine; AUC:Area under the curve; ROC:Receiver operating characteristic curve.

\section{Declarations}

\section{Ethics approval and consent to participate}

This study was approved by the Ethical Committee in Peking University Sixth Hospital and conducted based on the 1964 Declaration of Helsinki. All parents and children gave written consent before the onset of the experiment.

\section{Consent for publication}

The actor in the stimuli video signed a photo consent.

\section{Availability of data and materials}

The data that support the findings of this study are available on request from the corresponding author (Jing Liu). The data are not publicly available due to privacy or ethical restrictions.

\section{Competing interests}


The authors report no biomedical financial interests or potential conflicts of interest.

\section{Funding}

This study was supported by the National Key R\&D Program of China (Grant Number: 2017YFC1309900), Beijing Municipal \& Technology Commission (Grant Number: Z171100001017088), Beijing Natural Science Foundation (Grant Number: S170003), and Guangdong Key Project in "Development of new tools for diagnosis and treatment of Autism "( Grant Number: 2018B030335001).

\section{Authors' contributions}

$\mathrm{JL}, \mathrm{XLi}$ and $\mathrm{XLuo}$ developed the study concept. All authors contributed to the study design. Testing and data collection were performed by XG, TY and QW. QL and XG conducted the data processing and QW performed the statistical analysis under the supervision of $\mathrm{JL}$ and $\mathrm{LY}$. QL and QW drafted the paper, and $L Y, J L$ and XLi provided critical revisions. All authors approved the final version of the paper for submission.

\section{Acknowledgement}

We are grateful to the children and their parents for involvement in our study.

\section{References}

1. Delahaerche E, Chetouani M, Cohen D. Interpersonal synchrony: A survey of evaluation methods across disciplines and its application to ASD. Neuropsychiatrie de l'enfance et de l'adolescence. 2012;60(5).

2. Tony C. Commentary: Glass half full or half empty? Testing social communication interventions for young children with autism-reflections on Landa, Holman, O'Neill, and Stuart (2011). Journal of child psychology and psychiatry, and allied disciplines. 2011;52(1).

3. Fitzpatrick P, Romero V, Amaral JL, Duncan A, Barnard H, Richardson MJ, et al. Social Motor Synchronization: Insights for Understanding Social Behavior in Autism. J Autism Dev Disord. 2017;47(7):2092-107.

4. Condon WS, Sander LW. Neonate movement is synchronized with adult speech: interactional participation and language acquisition. Science. 1974;183(4120):99-101.

5. Condon WS, Sander LW. Synchrony demonstrated between movements of the neonate and adult speech. Child Dev. 1974;45(2):456-62.

6. Fitzpatrick P, Frazier JA, Cochran D, Mitchell T, Coleman C, Schmidt RC. Relationship Between Theory of Mind, Emotion Recognition, and Social Synchrony in Adolescents With and Without Autism. Front Psychol. 2018;9:1337.

7. Bernieri FJ, Davis JM, Rosenthal R, Knee CR. Interactional Synchrony and Rapport: Measuring Synchrony in Displays Devoid of Sound and Facial Affect. Pers Soc Psychol Bull. 1994;20(3):303- 
11.

8. Lakin JL, Chartrand TL. Using nonconscious behavioral mimicry to create affiliation and rapport. Psychol Sci. 2003;14(4):334-9.

9. Miles LK, Nind LK, Macrae CN. The rhythm of rapport: Interpersonal synchrony and social perception. Journal of Experimental Social Psychology. 2009;45(3).

10. Reddish P, Fischer R, Bulbulia J. Let's dance together: synchrony, shared intentionality and cooperation. PLoS One. 2013;8(8):e71182.

11. Reddish P, Bulbulia J, Fischer R. Does synchrony promote generalized prosociality? Religion Brain Behavior. 2014;4(1):3-19.

12. Wiltermuth SS, Heath C. Synchrony and cooperation. Psychol Sci. 2009;20(1):1-5.

13. Feldman R. Parent-infant synchrony and the construction of shared timing; physiological precursors, developmental outcomes, and risk conditions. J Child Psychol Psychiatry Allied Discip. 2007;48(34):329-54.

14. Hove MJ, Risen JL. It's All in the Timing: Interpersonal Synchrony Increases Affiliation. Social Cognition. 2009;27(6):949-60.

15. Shockley K, Richardson DC, Dale R. Conversation and Coordinative Structures. Topics in Cognitive Science. 2009;1(2suppl.):305-19.

16. Fitzpatrick P, Frazier JA, Cochran DM, Mitchell T, Coleman C, Schmidt RC. Impairments of Social Motor Synchrony Evident in Autism Spectrum Disorder. Front Psychol. 2016;7:1323.

17. Fitzpatrick P, Diorio R, Richardson MJ, Schmidt RC. Dynamical methods for evaluating the timedependent unfolding of social coordination in children with autism. Front Integr Neurosci. 2013;7:21.

18. Mundy P, Block J, Fau - Delgado C, Delgado C, Fau - Pomares Y, Pomares Y, Fau - Van Hecke AV, Van Hecke Av Fau - Parlade MV, Parlade MV. Individual differences and the development of joint attention in infancy. 2007(0009-3920 (Print)).

19. Mundy P, Jarrold W. Infant joint attention, neural networks and social cognition. 2010(1879-2782 (Electronic)).

20. Brooks R, Meltzoff AN. The development of gaze following and its relation to language. Dev Sci. 2005;8(6):535-43.

21. Dalton JC, Crais ER, Velleman SL. Joint attention and oromotor abilities in young children with and without autism spectrum disorder. J Commun Disord. 2017;69:27-43.

22. Mundy P, Newell L, Attention. Joint Attention, and Social Cognition. Curr Dir Psychol Sci. 2007;16(5):269-74.

23. Pickard KE, Ingersoll BR. Brief report: High and low level initiations of joint attention, and response to joint attention: differential relationships with language and imitation. J Autism Dev Disord. 2015;45(1):262-8.

24. Beuker KT, Rommelse NNJ, Donders R, Buitelaar JK. Development of early communication skills in the first two years of life. Infant Behavior Development. 2013;36(1):71-83. 
25. Vaughan Van Hecke A, Mundy PC, Acra CF, Block JJ, Delgado CE, Parlade MV, et al. Infant joint attention, temperament, and social competence in preschool children. Child Dev. 2007;78(1):53-69.

26. Nelson PB, Adamson LB, Bakeman R. Toddlers' joint engagement experience facilitates preschoolers' acquisition of theory of mind. Dev Sci. 2008;11(6):847-52.

27. Morales MMP. Individual differences in infant attention skills, joint attention, and emotion regulation behaviour. Int J Behav Dev. 2005;29(3):259-63.

28. Kim K, Mundy P. Joint attention, social-cognition, and recognition memory in adults. Front Hum Neurosci. 2012;6:172.

29. Oberwelland E, Schilbach L, Barisic I, Krall SC, Vogeley K, Fink GR, et al. Young adolescents with autism show abnormal joint attention network: A gaze contingent fMRI study. Neuroimage Clin. 2017;14:112-21.

30. American Psychiatric Association DSMTFAVAUS. Diagnostic and statistical manual of mental disorders: DSM-5 ${ }^{\text {TM }}$ (5th ed.). Codas. 2013;25(2):191.

31. Chawarska K, Klin A, Volkmar F. Automatic attention cueing through eye movement in 2-year-old children with autism. Child Dev. 2003;74(4):1108-22.

32. Falck-Ytter T, Fernell E, Hedvall AL, von Hofsten C, Gillberg C. Gaze performance in children with autism spectrum disorder when observing communicative actions. J Autism Dev Disord. 2012;42(10):2236-45.

33. Leekam SR, López B, Moore C. Attention and joint attention in preschool children with autism. Dev Psychol. 2000;36(2):261-73.

34. Vivanti G, Fanning PAJ, Hocking DR, Sievers S, Dissanayake C. Social Attention, Joint Attention and Sustained Attention in Autism Spectrum Disorder and Williams Syndrome: Convergences and Divergences. J Autism Dev Disord. 2017;47(6):1866-77.

35. Bedford R, Elsabbagh M, Gliga T, Pickles A, Senju A, Charman T, et al. Precursors to social and communication difficulties in infants at-risk for autism: gaze following and attentional engagement. J Autism Dev Disord. 2012;42(10):2208-18.

36. Falck-Ytter T, Thorup E, Bölte S. Brief report: Lack of processing bias for the objects other people attend to in 3-year-olds with autism. J Autism Dev Disord. 2015;45(6):1897-904.

37. Riby DM, Hancock PJ, Jones N, Hanley M. Spontaneous and cued gaze-following in autism and Williams syndrome. J Neurodev Disord. 2013;5(1):13.

38. Vivanti G, McCormick C, Young GS, Abucayan F, Hatt N, Nadig A, et al. Intact and impaired mechanisms of action understanding in autism. Dev Psychol. 2011;47(3):841-56.

39. Vivanti G, Trembath D, Dissanayake C. Atypical monitoring and responsiveness to goal-directed gaze in autism spectrum disorder. Exp Brain Res. 2014;232(2):695-701.

40. Gernsbacher MA, Stevenson JL, Khandakar S, Goldsmith HH. Why Does Joint Attention Look Atypical in Autism? Child Dev Perspect. 2008;2(1):38-45. 
41. Leekam S, Baron-Cohen S, Perrett D, Milders M, Brown S. Eye-direction detection: A dissociation between geometric and joint attention skills in autism. Br J Dev Psychol. 1997;15:77-95.

42. Fitzpatrick P, Romero V, Amaral JL, Duncan A, Barnard H, Richardson MJ, et al. Evaluating the importance of social motor synchronization and motor skill for understanding autism. Autism Res. 2017;10(10):1687-99.

43. Marsh KL, Isenhower RW, Richardson MJ, Helt M, Verbalis AD, Schmidt RC, et al. Autism and social disconnection in interpersonal rocking. Front Integr Neurosci. 2013;7:4.

44. Xavier J, Gauthier S, Cohen D, Zahoui M, Chetouani M, Villa F, et al. Interpersonal Synchronization, Motor Coordination, and Control Are Impaired During a Dynamic Imitation Task in Children With Autism Spectrum Disorder. Front Psychol. 2018;9:1467.

45. Senju A, Csibra G. Gaze following in human infants depends on communicative signals. Curr Biol. 2008;18(9):668-71.

46. Shockley K, Butwill M, Zbilut JP, Webber CL. Cross recurrence quantification of coupled oscillators. Physics Letters A. 2002;305(1).

47. Zbilut JP, Giuliani A, Webber CL. Detecting deterministic signals in exceptionally noisy environments using cross-recurrence quantification. Phys Lett A. 1998;246(1-2):122-8.

48. Shockley K, Santana MV, Fowler CA. Mutual interpersonal postural constraints are involved in cooperative conversation. Journal of Experimental Psychology Human Perception Performan. 2003;29(2):326-32.

49. Yu C, Smith LB. Joint attention without gaze following: human infants and their parents coordinate visual attention to objects through eye-hand coordination. PLoS One. 2013;8(11):e79659.

50. Auyeung B, Baron-Cohen S, Wheelwright S, Allison C. The Autism Spectrum Quotient: Children's Version (AQ-Child). J Autism Dev Disord. 2008;38(7):1230-40.

51. Li H, Liu J, Jia M, Yang L, Li X, Zhang A, et al. Reliability and validity of the Chinese version of Autism Spectrum Quotient-Children's version. Chinese Mental Health Journal. 2018;32(12):1000-5.

52. Kafai M, Eshghi K. CROification. Accurate Kernel Classification with the Efficiency of Sparse Linear SVM. IEEE Trans Pattern Anal Mach Intell. 2019;41(1):34-48.

53. Wang Q, Hoi SP, Wang Y, Lam CM, Fang F, Yi L. Gaze response to others' gaze following in children with and without autism. J Abnorm Psychol. 2020;129(3):320-9.

54. Elison JT, Paterson SJ, Wolff JJ, Reznick JS, Sasson NJ, Gu H, et al. White matter microstructure and atypical visual orienting in 7-month-olds at risk for autism. Am J Psychiatry. 2013;170(8):899-908.

55. Elsabbagh M, Fernandes J, Webb SJ, Dawson G, Charman T, Johnson MH. Disengagement of visual attention in infancy is associated with emerging autism in toddlerhood. Biol Psychiatry. 2013;74(3):189-94.

56. Moore A, Wozniak M, Yousef A, Barnes CC, Cha D, Courchesne E, et al. The geometric preference subtype in ASD: identifying a consistent, early-emerging phenomenon through eye tracking. Mol Autism. 2018;9:19. 
57. Wang Q, Hoi SP, Wang Y, Song C, Li T, Lam CM, et al. Out of mind, out of sight? Investigating abnormal face scanning in autism spectrum disorder using gaze-contingent paradigm. Dev Sci. 2020;23(1):e12856.

58. Yi L, Fan Y, Quinn PC, Feng C, Huang D, Li J, et al. Abnormality in face scanning by children with autism spectrum disorder is limited to the eye region: evidence from multi-method analyses of eye tracking data. J Vis. 2013;13(10).

59. Sasson NJ, Pinkham AE, Weittenhiller LP, Faso DJ, Simpson C. Context Effects on Facial Affect Recognition in Schizophrenia and Autism: Behavioral and Eye-Tracking Evidence. Schizophr Bull. 2016;42(3):675-83.

60. Cui X, Bryant DM, Reiss AL. NIRS-based hyperscanning reveals increased interpersonal coherence in superior frontal cortex during cooperation. Neuroimage. 2012;59(3):2430-7.

61. Wang Q, Han Z, Hu X, Feng S, Wang H, Liu T, et al. Autism Symptoms Modulate Interpersonal Neural Synchronization in Children with Autism Spectrum Disorder in Cooperative Interactions. Brain Topogr. 2020;33(1):112-22.

62. Reindl V, Gerloff C, Scharke W, Konrad K. Brain-to-brain synchrony in parent-child dyads and the relationship with emotion regulation revealed by fNIRS-based hyperscanning. Neuroimage. 2018;178:493-502.

63. Raven J, Court J, Jc R. Manual for Raven's Progresive Matrices and Vocabulary Scales, Sect. 3 Standard Progressive Matrices, 1996 Edition1983.

\section{Tables}

Table 1. Participant characterization

\begin{tabular}{|c|c|c|c|c|}
\hline Measure & ASD $(N=40)$ & TD $(N=40)$ & $P$ value & $t$ \\
\hline Male/Female ratio & $36 / 4$ & $36 / 4$ & - & - \\
\hline Age (in years) & $6.6 \pm 0.8$ & $6.7 \pm 0.6$ & 0.544 & -0.61 \\
\hline Non-verbal IQ ${ }^{\mathrm{a}}$ & $114.5 \pm 22.8$ & $119.3 \pm 18.1$ & 0.301 & -1.04 \\
\hline AQ-child ${ }^{b}$ & $85.8 \pm 8.6$ & - & - & - \\
\hline
\end{tabular}

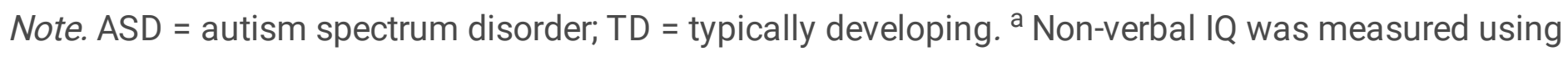
the Raven's Standard Progressive Matrices [63]. ${ }^{\mathrm{b}} \mathrm{AQ}$-child = the Autism Spectrum Quotient - Children's Version.

\section{Figures}


A.

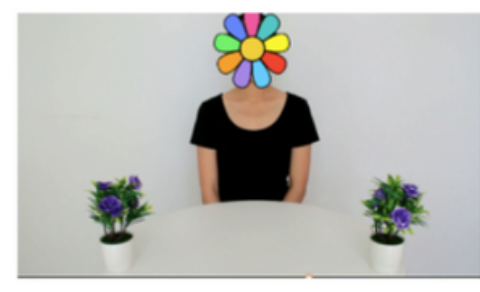

(a)

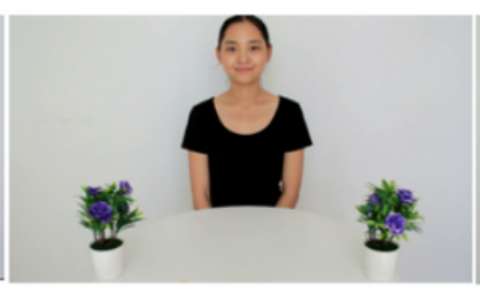

(b)

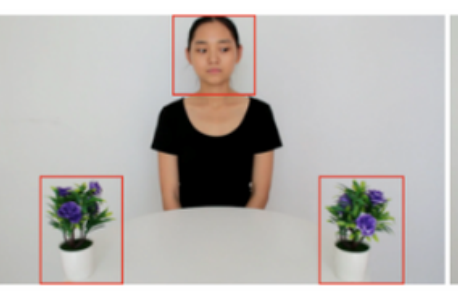

(c)

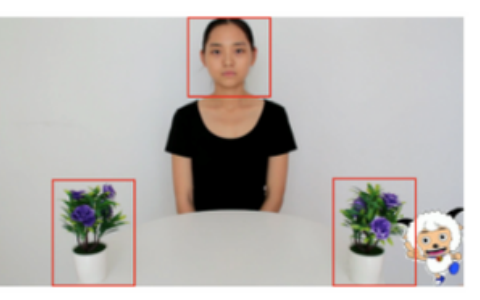

(d)

B.

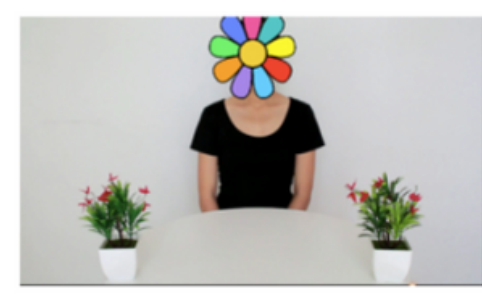

(a)

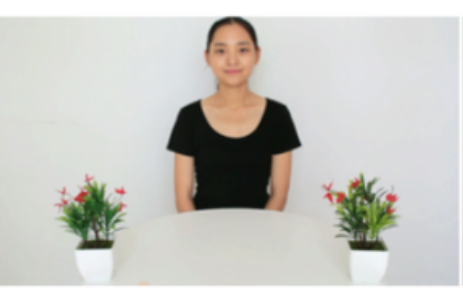

(b)
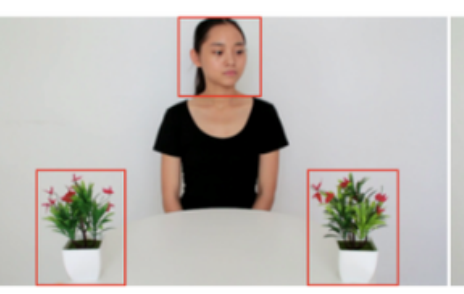

(c)

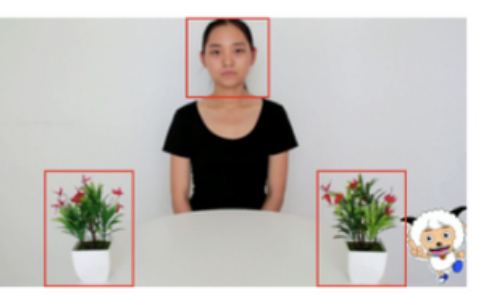

(d)

\section{Figure 1}

Screenshots of the video of the response to joint attention tasks. A and B were two rounds of the video where the objects on the table differed. (a) At the beginning of each round, the model's face was covered by a cartoon accompanied by a voiceover ('Look!') to attract the child's attention. (b) The model looks straight into the camera, smiling at the participant for about $5 \mathrm{~s}$. (c) The RJA task begins, the model shifts her gaze towards one of the two objects after looking straight into the camera for about $0.3 \mathrm{~s}$ and continues to look at the attended object for $4 \mathrm{~s}$. (d) The model turns back to face the camera for $3 \mathrm{~s}$ at the end of the task where a cartoon character appears beside the attended object. Areas of interests (AOI) are highlighted by red rectangles which do not appear in the video of RJA tasks. The person represented in the figure has permitted to publish the images in all formats. 


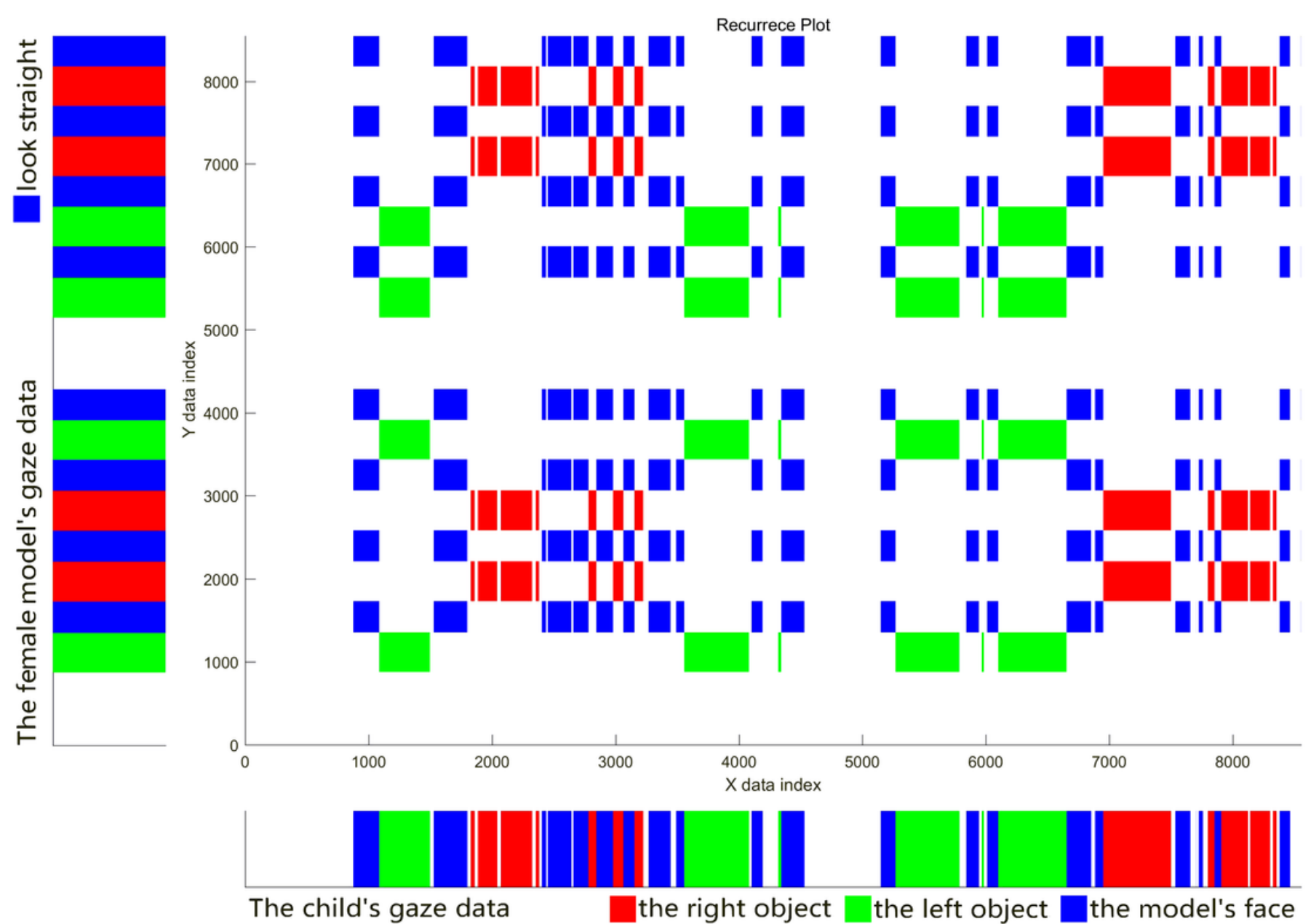

Figure 2

An example of cross-recurrence plot. The two gaze data streams at the left and bottom represent the AOls that the female model and the child were looking at over time, respectively. The colors at the center region represent the fact that the child and the female model were looking at the same object (the red or green pixels) or directly looking at each other (the blue pixels) at different time lags (the numbers at the $\mathrm{x}$ - and $y$-axes represent sample points, time unit: $1 / 120$ seconds). 
- ASD - TD - ASD rand level - TD rand level

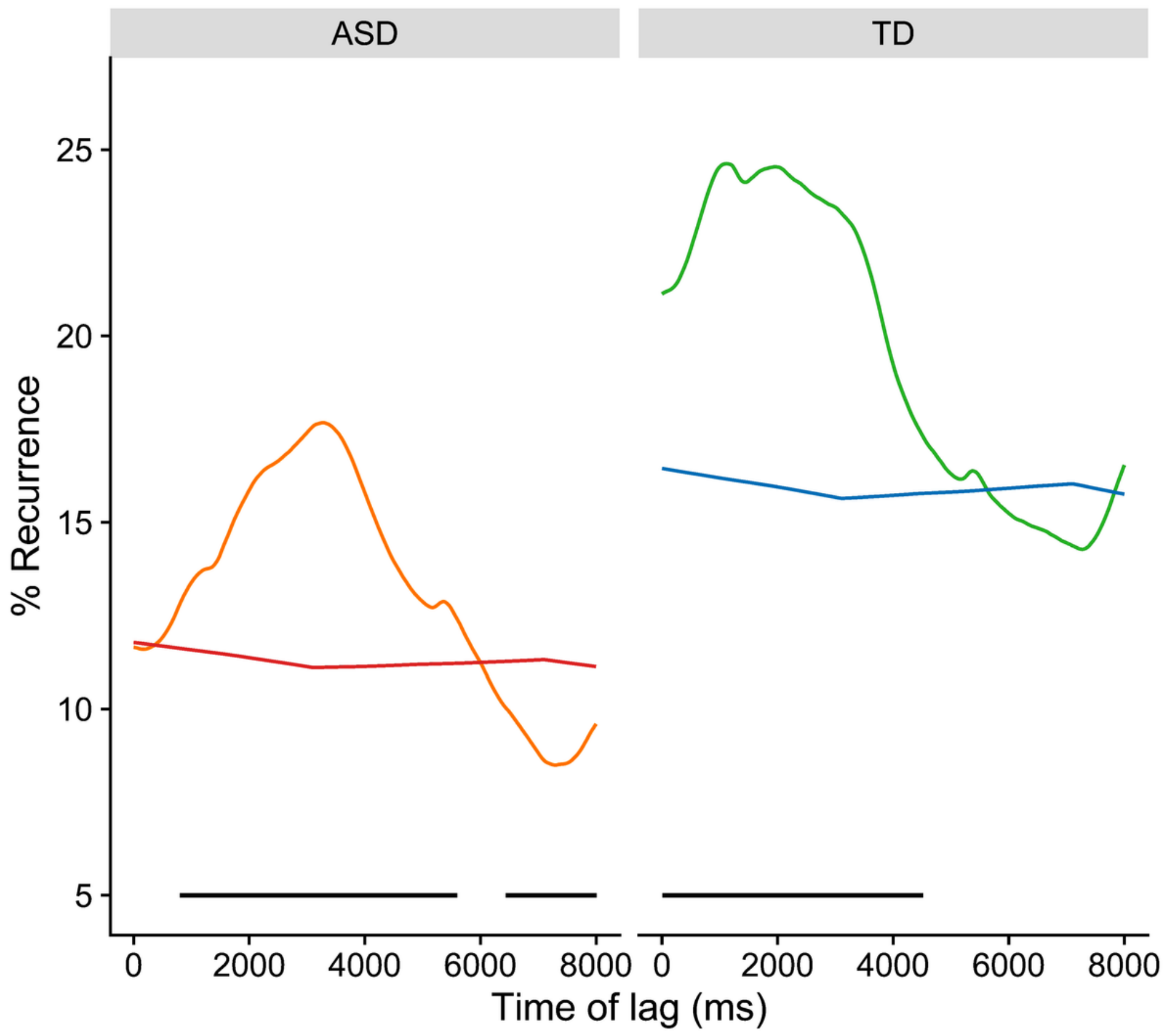

Figure 3

Average cross-recurrence at different time lags in the ASD and TD group pairings. The baseline of each group was generated by shuffling the temporal order of each child's eye-movement sequence 1000 times and calculating the average percentage recurrence with the female model for each child, and then averaging the percentage recurrence across the children for each group. The black line below indicated the lag time at which the child and the female model were looking at the same AOI at above-chance levels. 


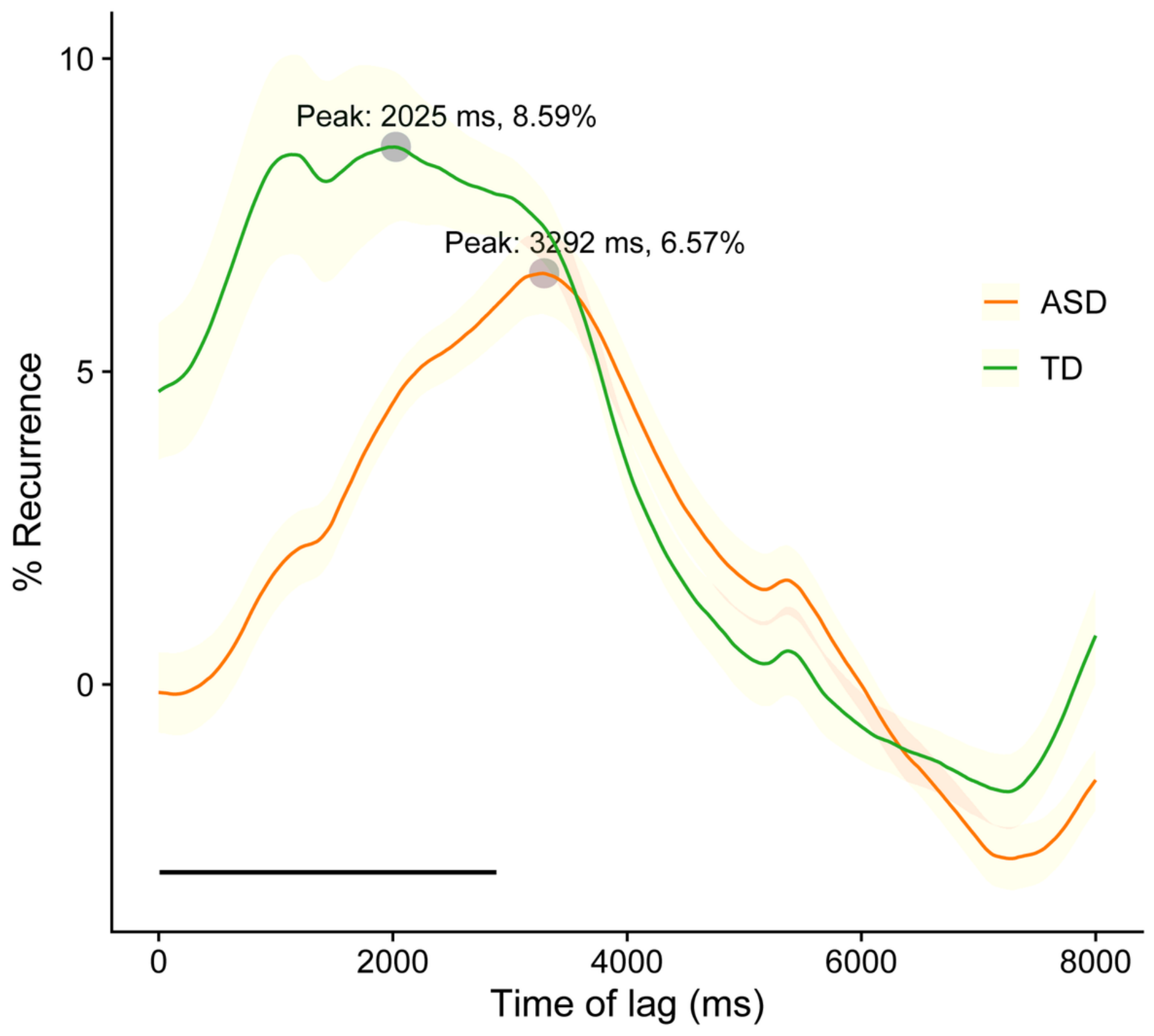

Figure 4

The comparison of average cross-recurrence between the ASD and TD group pairing with the baseline adjusted. The black line below indicates the lag time at which the average percentage recurrence in the ASD and TD group were significantly different.

\section{Supplementary Files}

This is a list of supplementary files associated with this preprint. Click to download. 
- Supplementarymaterials.docx

Page 21/21 